\title{
7 - Effects of age, gender, and response time on the outcomes of patients following out-of-hospital cardiac arrest in Asia
}

Jiashan Chen ${ }^{1}$, Wen-Chu Chiang ${ }^{2}$, Jen-Tang Sun ${ }^{1}$, Chih-Wei yang ${ }^{2}$, Hui-Chih Wang ${ }^{2}$, Ying-Chih Ko ${ }^{2}$, Matthew Huei-Ming Ma

1 Department of Emergency Medicine, Far Eastern Memorial Hospital, New Taipei City, Taiwan 2 Department of Emergency Medicine, National Taiwan University Hospital, Taipei, Taiwan

\section{Background}

Out-of-hospital cardiac arrest (OHCA) is one of the major public health concerns with, e.g., mortality rates $7.5 \%$ in the United States and $10.8 \%$ in Denmark. In the recent 5 years, the trend of survival rates has overall increased by $2 \%$.

However, the survival rate of OHCA patients in Asia nowadays is still only 5.4\%, remaining relatively low compared with the western countries. Many prehospital factors have proved to be associated with the survival rate of OHCA, including initial cardiac rhythm, bystander CPR, bystander witnessed, location, and EMS system factors. However, whether gender and age are associated with the survival rate of OHCA remains controversial. While there have been Asian studies showing gender disparity in the survival rates of OHCA (women had better outcomes than men), similar studies in the western countries presented totally opposite results.

\section{Aim}

This study aims to identify whether gender and age factors affect the survival rate of OHCA.

\section{Method}

We analyzed the Utstein-based registry OHCA data from PanAsian Resuscitation Outcomes Study (PAROS) from 2009 to 2012.

Inclusion criteria: adult, aged $\geqq 18$ years, non-traumatic, underwent resuscitation attempts.

Exclusion criteria: witnessed by EMT, response time $\geqq 60$ minutes.

Subgroup analysis: young man $(<65$ years old), elder man ( $\geq 65$ years old), young women $(<65$ years old), and elder women ( $\geq 65$ years old).

Exposures: response time

Outcome of interest: survival to discharge

\section{Results}

Enrolled patients: 50,276 patients

Main findings:

- Increased response time was negatively associated with the survival rate (aOR 0.93, 95\% CI $0.91-0.94$ )

- Survival chance decreased by $7 \%$ per minute increase of response time.

Subgroup analysis: the young male group had the highest crude survival rate, followed by the young female, elder male, and elder female groups (Figure 1). However, after adjusting multiple factors*, young female became the top survival group, followed by the young male, elder male and elder female groups (Figure 2).

*witnessed status, shockable rhythm, bystander cardiopulmonary resuscitation, and prehospital advanced life support

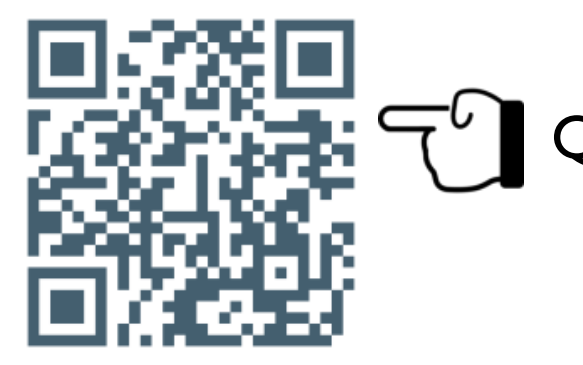

Questions? Scan it to contact the author! Jiashan Chen ccjia3@gmail.com

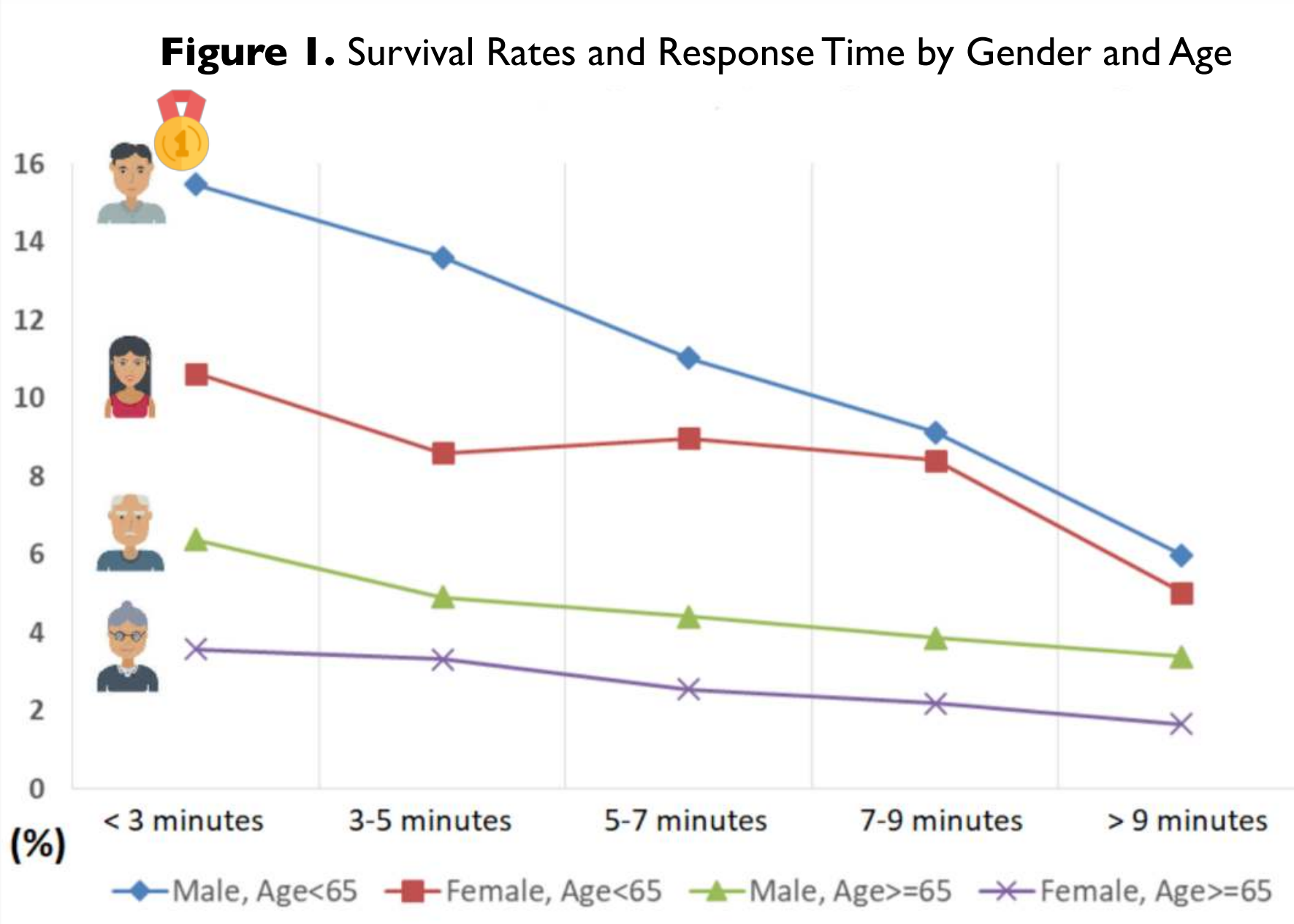

Figure 2. Adjusted Odds Ratio in Logistic Regression

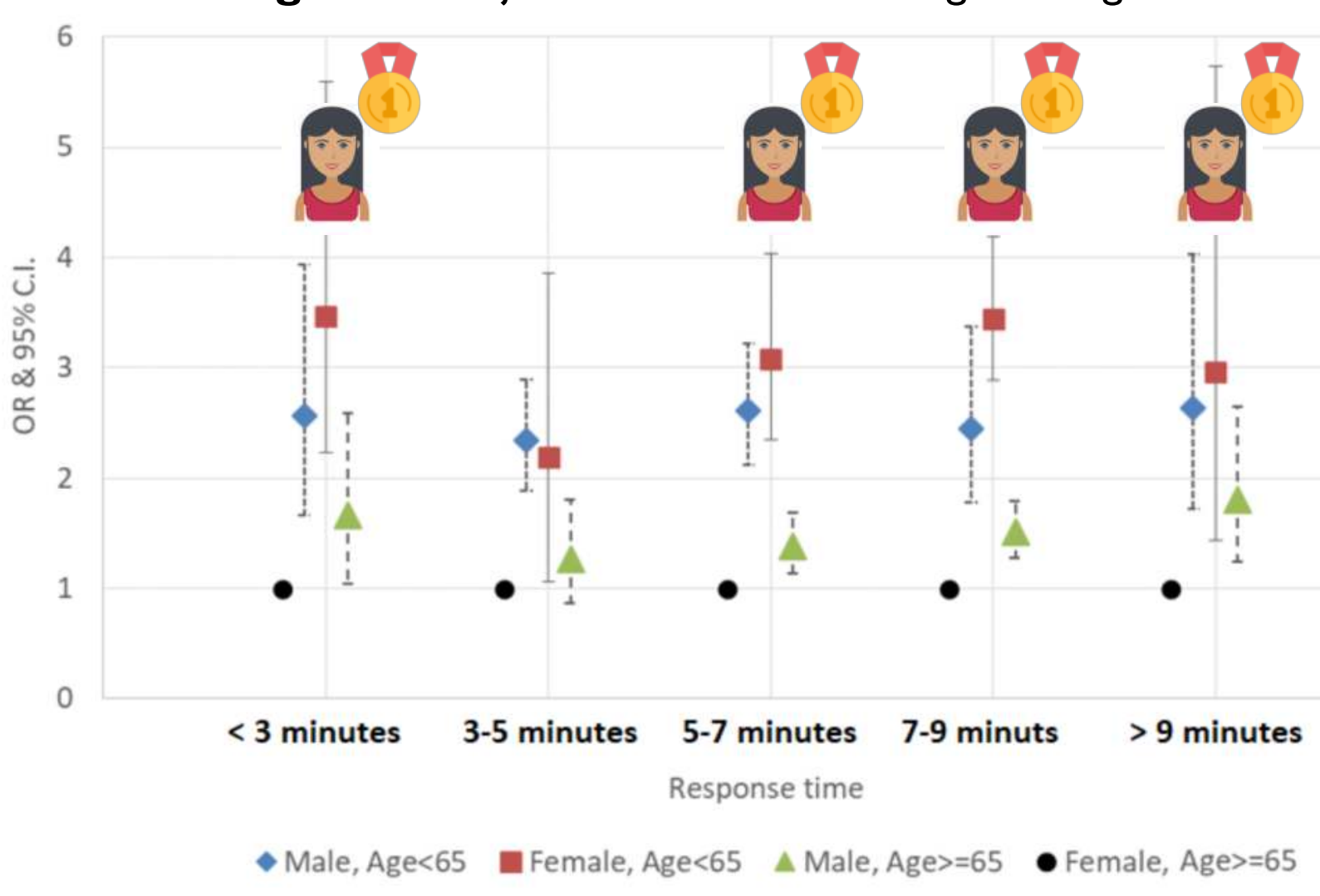

Conclusions

- The chance of OHCA survival to discharge decreased 7\% by per minute increase of response time in Asia.

- Although young male after OHCA appeared to have the highest survival rates, young female might have better chances of survival compared to young male if received the same prehospital attention and intervention. 